\title{
Experimental observations of modes with geodesic acoustic character from the core to the edge in the TCV tokamak
}

\author{
Zhouji Huang, Stefano Coda, Gabriele Merlo, Stephan \\ Brunner, Laurent Villard, Benoit Labit, Christian Theiler, \\ and the TCV team $\ddagger$ \\ Swiss Plasma Center, Ecole polytechnique Fédérale de Lausanne, CH-2015 \\ Lausanne, Switzerland \\ E-mail: zhouji.huang@epfl.ch
}

\begin{abstract}
The geodesic acoustic mode (GAM) is a coherently oscillating mode, related to the zonal flows that can regulate turbulence in magnetized toroidal plasmas. Modes possessing geodesic acoustic character have been widely observed in the TCV tokamak. A transition has been observed in the course of a single discharge from a continuum regime to a radially extended single-frequency regime. The mode has been also observed and characterized for the first time in the scrapeoff layer, primarily by Langmuir probes, suggesting a particle flow to the edge modulated at the mode frequency. These experimental observations are consistent with nonlinear global gyrokinetic simulations, reported in a companion paper [1]. These also suggest a possible coupling with radial avalanche phenomena.
\end{abstract}

\section{Introduction}

Zonal flows are azimuthally symmetric band-like sheared flows that are widely observed in nature and in the laboratory $[2,3]$. In the context of magnetized toroidal plasmas, zonal flows are sheared $n=0, m=0 E \times B$ flows associated with an electric field fluctuation which is constant on magnetic surfaces but varies perpendicularly with finite radial wave number. Zonal flows are excited nonlinearly by all types of microinstabilities and in turn regulate transport by shearing the turbulent eddies. Understanding this self-organization mechanism is an important issue in magnetic confinement fusion research.

The geodesic acoustic mode (GAM) [4] is a finite frequency branch of zonal flows coupled to a $n=0, m=1$ up-down-antisymmetric pressure perturbation in a toroidal plasma. The GAM frequency is predicted to depend on the ratio of the ion sound speed $c_{\mathrm{s}} \approx \sqrt{\left(T_{\mathrm{e}}+T_{\mathrm{i}}\right) / m_{\mathrm{i}}}$ to the major radius $R: f=F \sqrt{2+q^{-2}} c_{\mathrm{s}} /(2 \pi R)$. Here, $T_{\mathrm{e}}$ and $T_{\mathrm{i}}$ are the electron and ion temperatures, respectively, $m_{\mathrm{i}}$ is the ion mass, $q$ is the safety factor, $F$ is a coefficient of order unity which depends on plasma and shape parameters (especially elongation) [5, 6, 7]. The radial distribution and dispersion of GAMs differ between experiments. A continuum of GAM spectra has long been reported in many tokamaks [8]; in this case the mode frequency depends on radius according

$\ddagger$ See author list of S. Coda et al 2017 Nucl. Fusion 57102011 
to the local analytical formula and following the radial variation of the temperature. In other cases however, radial eigenmodes are also observed, i.e., a mode frequency that stays constant over a significant radial extent. In some cases there are several discrete coexisting eigenmodes [5, 9], whereas in others a single-frequency $[10,11,12]$ is observed. Analytical work [13] has shown that finite Larmor radius effects lead to the existence of global GAM eigenmodes, which could potentially explain the observations. Analytical work based on MHD [14] also found conditions for the existence of a global GAM. However, for the TCV experiments reported here, the single frequency regime is observed at frequencies below the local GAM frequency, whereas the above-mentioned analytical works predict a global GAM propagating at frequencies above the local GAM frequency. Other possible explanations include a radially extended dominant component associated with the continuum GAM [15]. In this paper, we present an experimental observation of a regime transition between continuum and singlefrequency in an electron-cyclotron-heated limited L-mode discharge in the course of a plasma current ramp-up in TCV.

Oscillations at the edge GAM frequency also appear in multiple other fluctuating fields. In the 2011-2013 TCV campaign [11], GAMs were simultaneously observed as a coherent mode in four different fields: the $m=0 \tilde{E} \times B$ flow, the $m=1$ density component $\tilde{n}$, the electron temperature component $\tilde{T}_{\mathrm{e}}$, as well as the magnetic component $\tilde{B}_{\theta}$, which was found to have toroidal mode number $n=0$ and poloidal mode number dominated by $m=2$, consistent with theoretical predictions [16]. The magnetic component of the GAM was also reported from the DIII-D [9], T-10 [12] and Globus-M [17] tokamaks. In the latter case oscillations at the GAM frequency were also observed on the $D_{\alpha}$ emission intensity. In the 2016 TCV campaign, oscillations at the edge GAM frequency were detected in divertor L-mode discharges for the first time by several scrape-off layer (SOL) diagnostics: magnetic probes (pickup coils) and Langmuir probes near the strike points, and also $D_{\alpha}$ emission signals. GAM-like divertor fluctuations were also observed on inner and outer divertor probes in I-mode regime in Alcator C-Mod [18].

The remainder of this paper is organised as follows. The experimental setup is presented in section 2 and the identification of the mode is discussed in section 3. Section 4 reports on the transition from a continuum to a global mode. A characterization of the oscillations at the edge GAM frequency in the SOL is presented in section 5 , followed by conclusions in section 6 .

A companion paper [1] presents an extensive theoretical and numerical analysis of these modes in TCV.

\section{Experimental setup}

The experiments were performed on the TCV tokamak [19, 20] at the Swiss Plasma Center, EPFL, Switzerland. The major radius and minor radius are $0.88 \mathrm{~m}$ and $0.25 \mathrm{~m}$, respectively. The GAMs were studied in Ohmic and L-mode deuterium plasmas with toroidal magnetic field $B_{\mathrm{T}} \cong 1.3 \mathrm{~T}$, plasma current $I_{\mathrm{p}}=100-350 \mathrm{kA}$, central line-integrated electron density $n=0.5-5 \times 10^{19} \mathrm{~m}^{-2}$, and in both limited and diverted configurations. The divertor configurations include lower single-null (LSN) topologies with different levels of poloidal and total flux expansion (including X- and Super-X divertors [21]), as well as upper single null (USN), double null (DN), and snowflake $[22,23]$ (SF) topologies, made possible by the unique shaping capability of the TCV tokamak. The auxiliary heating is applied in the form of electron cyclotron 
resonance heating (ECRH) at the second (X2) harmonic with up to $1.35 \mathrm{MW}$ input power.

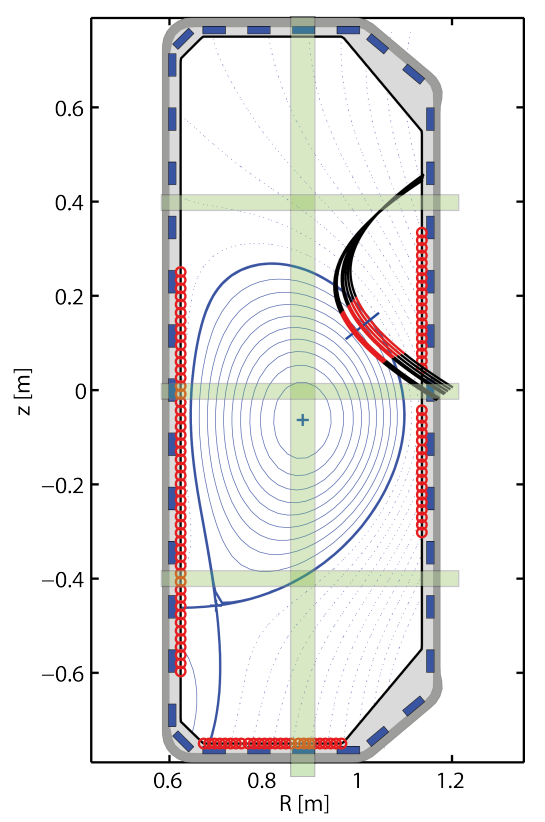

Figure 1. Schematic figure of the main diagnostics used in this paper on TCV. The black lines at the top right represent the poloidal projections of the TPCI laser chords, the red lines highlight the spatial selected integration lengths. The red circles and blue squares represent the Langmuir probes and the magnetic probes, respectively. The green rectangular box represents the $D_{\alpha}$ line-of-sights.

A schematic figure of the main diagnostics used in this paper is shown in Fig. 1. The main tool used to study the GAM regime transition is the tangential phase contrast imaging (TPCI) diagnostic [24], a laser-based system measuring line-integrated electron density fluctuations, with radial spatial resolution enhanced by the tangential injection geometry using spatial filtering techniques: for a radial mode such as the density component of the GAM, the TPCI measurement is localized at the point where the laser is tangent to the magnetic flux surfaces. The diameter of the laser beam is $5 \mathrm{~cm}$, subdivided by the detection geometry into 9 parallel and co-planar chords. The entire radial distribution of the GAM can be resolved by shifting the plasma column vertically in the highly-elongated TCV vacuum vessel. The present frequency bandwidth is $1.6 \mathrm{MHz}$ and wave number resolution is in the range of ion scale microinstabilities, $1 \mathrm{~cm}^{-1}<k_{\rho}<9 \mathrm{~cm}^{-1}$.

The magnetic component of the GAM is measured by the magnetic probe arrays installed on the inside wall of the device. Each probe is absolutely calibrated and measures the time derivative of the magnetic field component along the probe axis, which lies in a poloidal plane and is aligned with the vessel wall [25]. Three pairs of toroidal arrays are installed at the mid-plane of the vacuum vessel, and $35 \mathrm{~cm}$ above and below it, respectively. Each pair includes 8 magnetic probes on the high field side (HFS) and 16 probes on the low field side (LFS) located at equidistant toroidal angles, allowing the identification of toroidal mode number $n=0-8$. Four poloidal arrays at four poloidal cross sections toroidally separated by $90^{\circ}$ are each equipped with 38 
near-evenly spaced magnetic probes, providing a high-resolution measurement of the poloidal mode number $m$ of modes inside the last closed flux surface (LCFS), as well as localized features in the SOL e.g., near strike points in a divertor configuration. The main SOL diagnostic is a set of 114 wall-mounted Langmuir probes, located on the floor and both the HFS and LFS walls of the vacuum vessel. The Langmuir probe set allows measurements in many geometries, such as LSN divertors with strike points moving broadly from the HFS to the LFS and with varying poloidal flux expansion, and SF plasmas. Langmuir probe measurements for the upper strike points in the USN and DN configurations are not available. Each Langmuir probe is biased to a constant $-100 \mathrm{~V}$ to measure ion saturation currents. The final diagnostic is a set of $D_{\alpha}$ emission photodiodes, one viewing vertically from the top, and three viewing horizontally from the LFS to $z= \pm 40 \mathrm{~cm}$ and $z=0 \mathrm{~cm}$ (the midplane), respectively.

\section{Mode identification}

The characteristics of these oscillations are found to be consistent with the standard GAM in three different respects: frequency scaling, axisymmetry and mode-turbulence interactions, as illustrated in Fig. 2. Figure 2 (a) shows the spectrogram of a magnetic probe signal in an Ohmic discharge featuring a density ramp, at the outer strike point on the vessel floor. The GAM frequency scales with the square root of the plasma temperature, and thus decreases during the Ohmic density ramp-up. As in this case the GAM appears as a radially extended mode, the calculated frequency uses $T_{\mathrm{e}}$ at $\rho_{\psi}=0.95$, which is usually the outer limit of the GAM radial range. The toroidal mode number is found to be $n=0$ from the bottom toroidal magnetic probe array at the HFS, as shown in Fig. 2 (b) and (c). Finally, Figs. 2 (d) and (e) illustrate the strong bicoherence at the GAM frequency and broadband turbulence frequencies from magnetic measurement, indicating nonlinear coupling between the mode and the turbulence. Therefore these modes are identified as GAMs.

\section{Study of GAM regime transition}

A regime transition between continuum and single-frequency is observed in a limited L-mode discharge with electron-cyclotron heating $(\mathrm{ECH})$ in the course of a plasma current ramp-up. An overview of the plasma parameters for this shot is shown in Fig. 3. The Thomson scattering system measures a central electron density $n \approx 4 \times 10^{19} \mathrm{~m}^{-3}$, while the edge electron density at $\rho_{\psi}=0.95$ from $t=0.7$ to $1.3 \mathrm{~s}$ is around $1.2 \times 10^{19} \mathrm{~m}^{-3}$; the electron temperature $T_{\mathrm{e}}$ increases from 600 to $900 \mathrm{eV}$ in the center while remaining relatively constant around $150 \mathrm{eV}$ at $\rho_{\psi}=0.95 ; I_{\mathrm{p}}$ increases from 100 to $180 \mathrm{kA}$ during the transition, corresponding to a $q_{95}$ ramp-down from 8 to 5.5 ; the shape of the plasma is nearly constant during the ramp, with edge elongation $\kappa=1.52$ and triangularity $\delta=0.5-0.6$. Three X2 ECH beams, each with $450 \mathrm{~kW}$ power, are injected into the plasma and the resonance layer is at $\rho_{\psi}=0.5$. The ECH power is absorbed outside the $q=2$ rational surface before $t=0.9 \mathrm{~s}$ and inside the $q=2$ surface afterwards.

The transition from the GAM continuum to a radially extended regime starts at about $t=0.9 \mathrm{~s}$. The radial profile of the GAM during the transition is illustrated in Fig. 4, measured by 8 parallel chords of the TPCI system. The radial range of the measurement slightly changes with time due to a shift of plasma vertical position. At $t=0.7-0.9 \mathrm{~s}$, the GAM appears mainly as a frequency continuum with multiple 

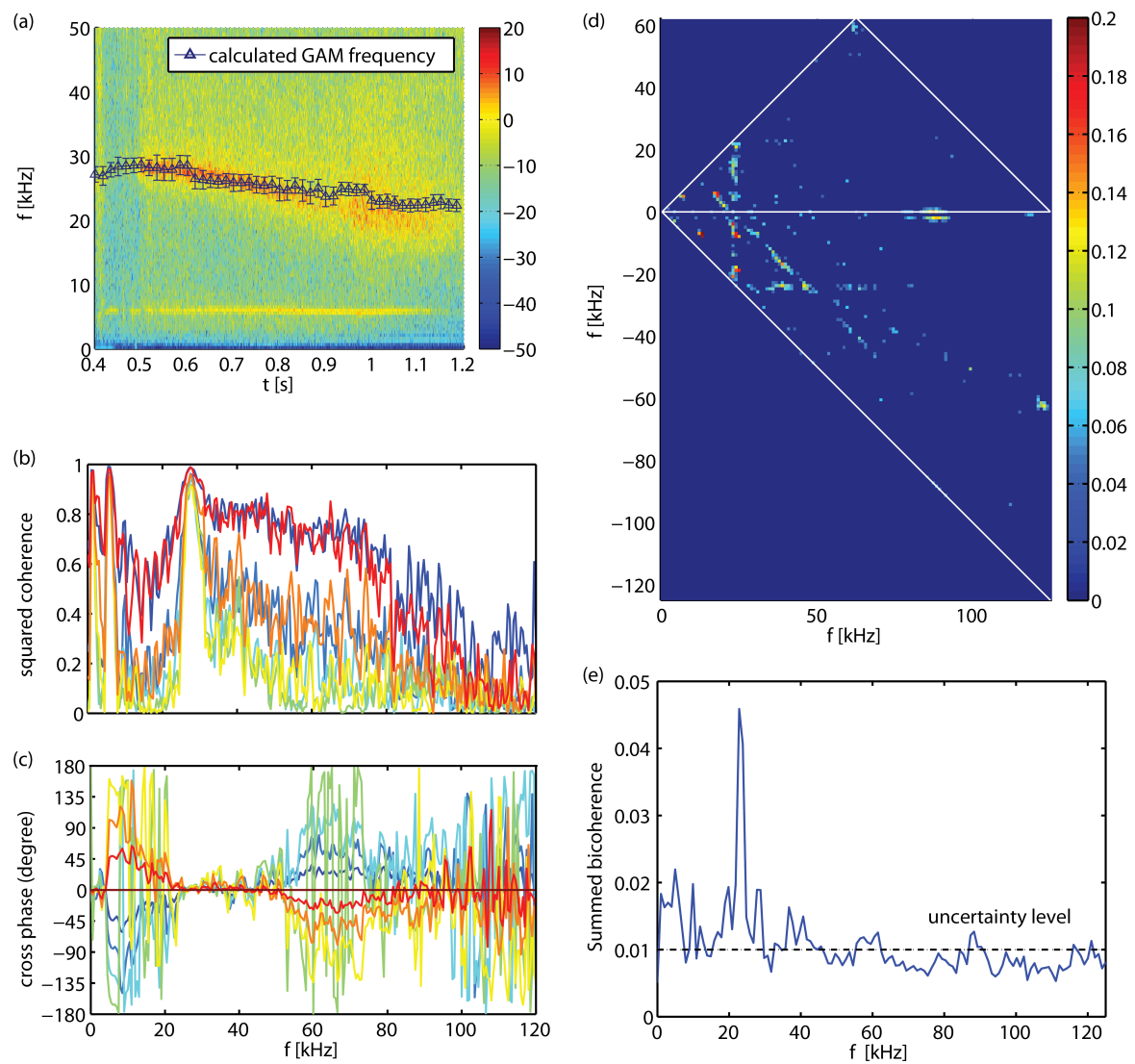

Figure 2. (a) Auto-power spectrogram of magnetic fluctuations at outer strike point in TCV discharge 52020 featuring a density ramp; the triangle markers indicate the analytical GAM frequency using $T_{\mathrm{e}}$ at $\rho_{\psi}=0.95$. (b),(c) Squared coherence and cross phase of magnetic fluctuations between a reference magnetic probe and the other 7 in the toroidal HFS bottom array in TCV discharge 54490, calculated over a $40 \mathrm{~ms}$ time window with $0.5 \mathrm{kHz}$ frequency resolution. (d),(e) Squared bicoherence (only points with bicoherence larger than the statistical uncertainty level are shown) and summed bicoherence of magnetic fluctuations at outer strike point in TCV discharge 54490, calculated over a $100 \mathrm{~ms}$ time window with $1 \mathrm{kHz}$ frequency resolution.

distinguishable eigenmodes, with the overall frequency decreasing with the radial location. From $t=0.9 \mathrm{~s}$ to $1.1 \mathrm{~s}$, a mode at $27 \mathrm{kHz}$ starts to dominate outside $\rho_{\psi}=0.74$, with another, weaker, coexisting mode at $28.4 \mathrm{kHz}$; in the region $\rho_{\psi}<0.74$ the mode frequency is still dependent on radius. Finally at $t=1.1-1.3 \mathrm{~s}$, the GAM has fully developed into a coherent structure at $27.6 \mathrm{kHz}$ over a radial range from $\rho_{\psi}=0.72$ to the outermost position of the measurement at $\rho_{\psi}=0.85$. Another $29.4 \mathrm{kHz}$ mode can still be observed only on the innermost chord. The measured frequency is always lower than the local analytical GAM frequency (the dashed line in Fig. 4), which is calculated with the simplified dispersion relation provided in Ref. [7] accounting the plasma elongation $\kappa$ :

$$
f=\sqrt{\frac{2}{1+\kappa^{2}}} \sqrt{2+1 / q^{2}} c_{s} / R
$$


TCV discharge \#48068
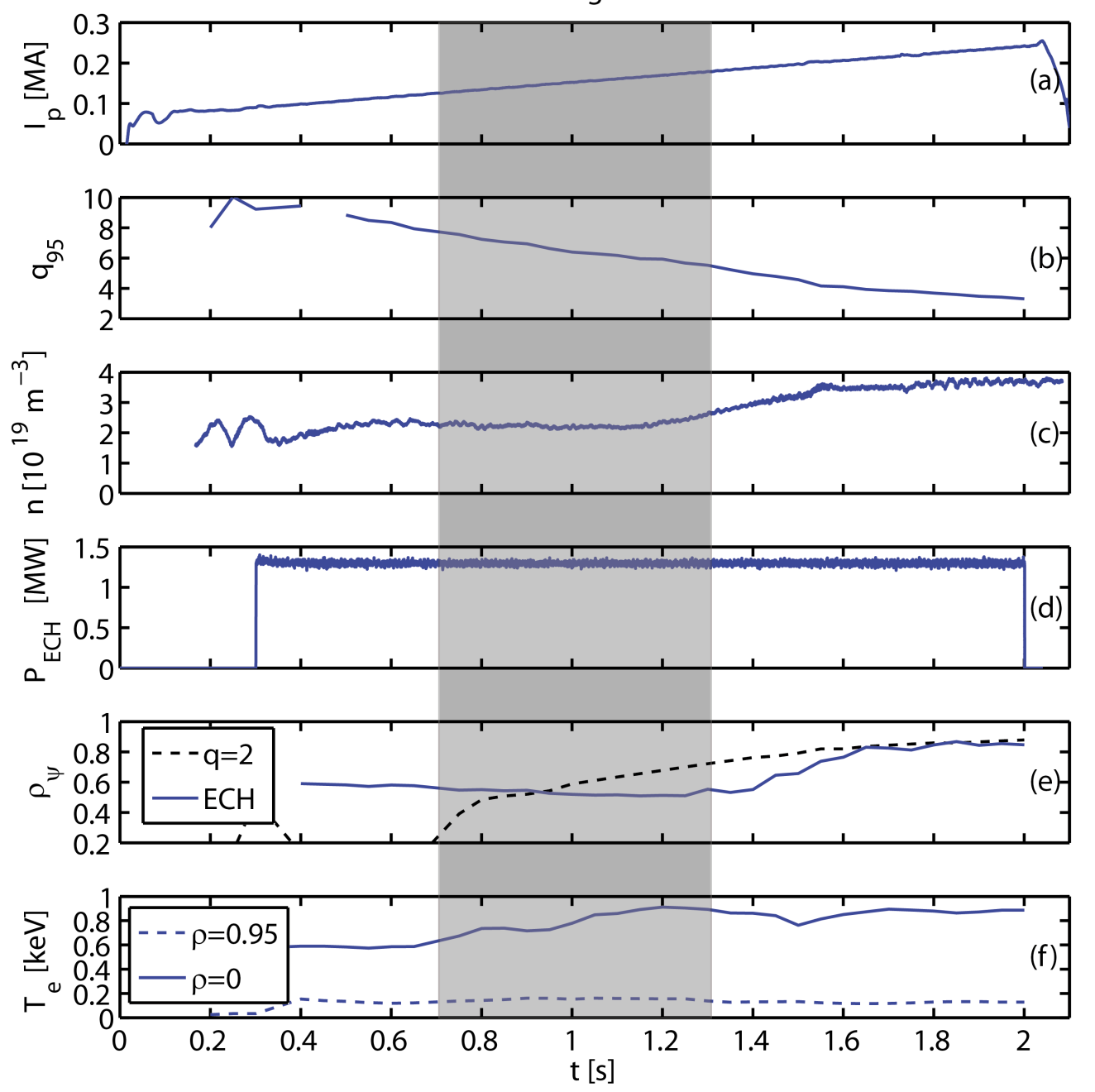

Figure 3. Time traces of (a) plasma current, (b) safety factor at $95 \%$ of minor radius $\rho_{\psi}$ (normalized square root of the poloidal magnetic flux), (c) line-averaged electron density from far infrared interferometer, (d) total ECH input power, (e) radial location of $q=2$ surface and ECH absorption layer, (f) electron temperature measured by Thomson scattering at $\rho_{\psi}=0$ and 0.95 in TCV discharge 48068. The shaded region indicates the time window for GAM regime transition analysis.

In this discharge the $T_{\mathrm{i}}$ measurement from CXRS is not available, an empirical ratio of electron to ion temperature $T_{\mathrm{i}} / T_{\mathrm{e}}=0.66$ for similar discharges was used in the calculation. Note that the mode frequency tends to match the analytical prediction at the outermost radial position. Spatial correlation analysis on 9 radially separated TPCI chords shows that the radial wave number of the GAM is $k_{\rho}=2 \mathrm{~cm}^{-1}$, outward propagating, which is consistent with previous observations of single-frequency radially coherent GAMs on TCV [11].

Local and global nonlinear gyrokinetic simulations have been performed with 

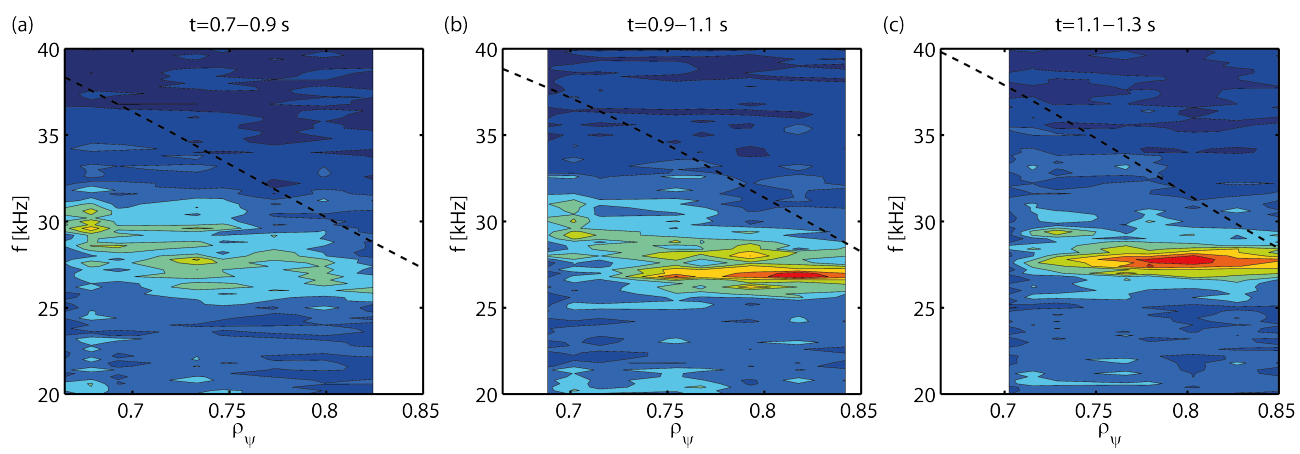

Figure 4. Auto-power spectrum of electron density fluctuations from TPCI measurement with $0.2 \mathrm{kHz}$ frequency resolution in TCV discharge 48068. The dashed line indicates the analytical local GAM frequency.

GENE [26] with reference $q, n_{\mathrm{e}}, T_{\mathrm{e}}$ and $T_{\mathrm{i}}$ profiles from TCV experimental plasmas of both GAM continuum and radially coherent mode cases, as reported in the companion paper [1]. Nonlinear flux-tube simulations show that the $E \times B$ velocity oscillation frequency is always dependent on the local temperature with both reference profiles, however downshifted from the local analytical GAM frequency. Results from nonlinear global simulations on the other hand are fairly consistent with experimental observations: a coherent, mostly outward propagating, single-frequency oscillation of the flux surface averaged $E \times B$ velocity is found to exist over the radial range from $\rho_{\psi}=0.65$ to the simulation boundary, with $T, n$ and $q$ profiles from the radially extended GAM time slice; while the GAM frequency follows the local RosenbluthHinton prediction [27] with profiles from the GAM continuum time slice [1].
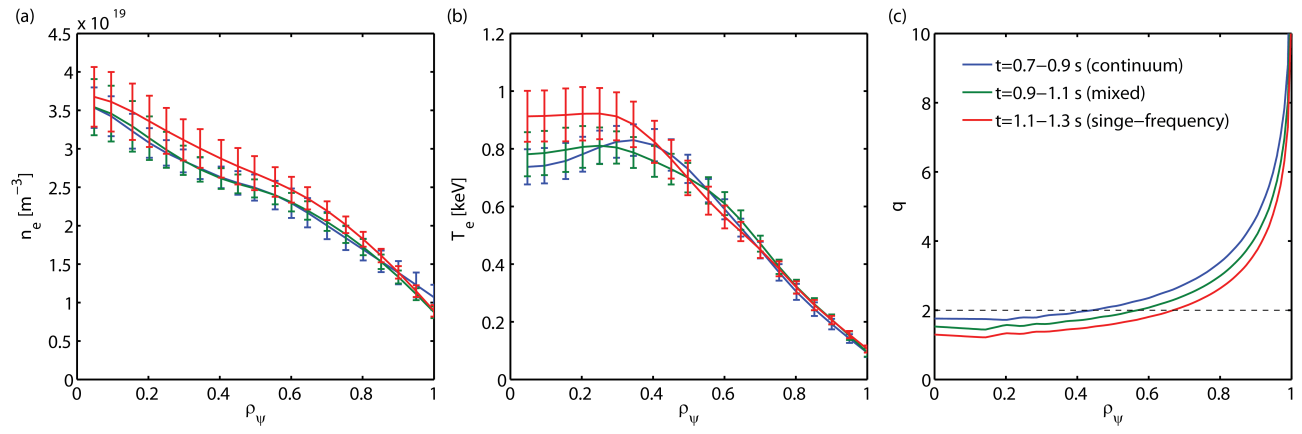

Figure 5. Electron density (a), temperature (b) and safety factor (c) profiles of TCV discharge \#48068 over the time range of GAM regime transition.

The origin of the different GAM regimes can potentially be traced to the different $q$ profiles, but also the different electron temperature and density as well as the corresponding gradient profiles, which would affect the turbulence driving the GAM. Also, in this discharge the ECH deposition position was passing the $q=2$ surface at $t=0.9 \mathrm{~s}$ when the transition from continuum to radially extended mode started; however, the overall $T_{\mathrm{e}}$ and $n_{\mathrm{e}}$ profiles only exhibit a distinguishable increase after $t=1.1 \mathrm{~s}$, as shown in Fig. 5. In order to distinguish the two effects, another global nonlinear GENE run was carried out with mixed conditions: $T_{\mathrm{e}}$ and $n_{\mathrm{e}}$ profiles from 
the radially extended GAM time slice, and $q$ profile from the continuum GAM time slice. The results show that in this case the GAM is in the radially extended regime. In contrast, the results from the simulation with reversed conditions, i.e., $T_{\mathrm{e}}$ and $n_{\mathrm{e}}$ profiles from the continuum GAM discharge and $q$ profile from the radially extended GAM time slice, show that the GAM is in the continuum regime [1]. Additionally, it is shown in fig. 5 that the GAM regime transition starts prior to the change in $T_{\mathrm{e}}$ and $n_{\mathrm{e}}$ profiles. Therefore, the conclusion is that varying the safety factor is not the cause behind the transition from continuum to radially extended regime.

\section{GAMs in the scrape-off layer}

In the 2016 TCV campaign, oscillations at the edge GAM frequency were detected in divertor L-mode discharges by several scrape-off layer (SOL) diagnostics: magnetic probes (pickup coils) and Langmuir probes near the strike points, and also $D_{\alpha}$ emission signals. In order to understand these oscillations, multi-diagnostic measurements have once again been performed: TPCI is employed for measurement of conventional GAMs inside the LCFS; the $\tilde{J}_{\mathrm{i} \text {,sat }}$ component is measured at the strike points by Langmuir probes; the $\dot{\tilde{B}}_{\theta}$ component can be detected on magnetic probes at the two conventional strong $m=2$ anti-nodes at the HFS wall, as well as at the strike points, as shown in Fig. 6; on $D_{\alpha}$ emission, oscillations are observed mainly along the vertical line-of-sight channel, but also sometimes on the lateral line-of-sight channel in the bottom part of the vessel when the mode is sufficiently strong.
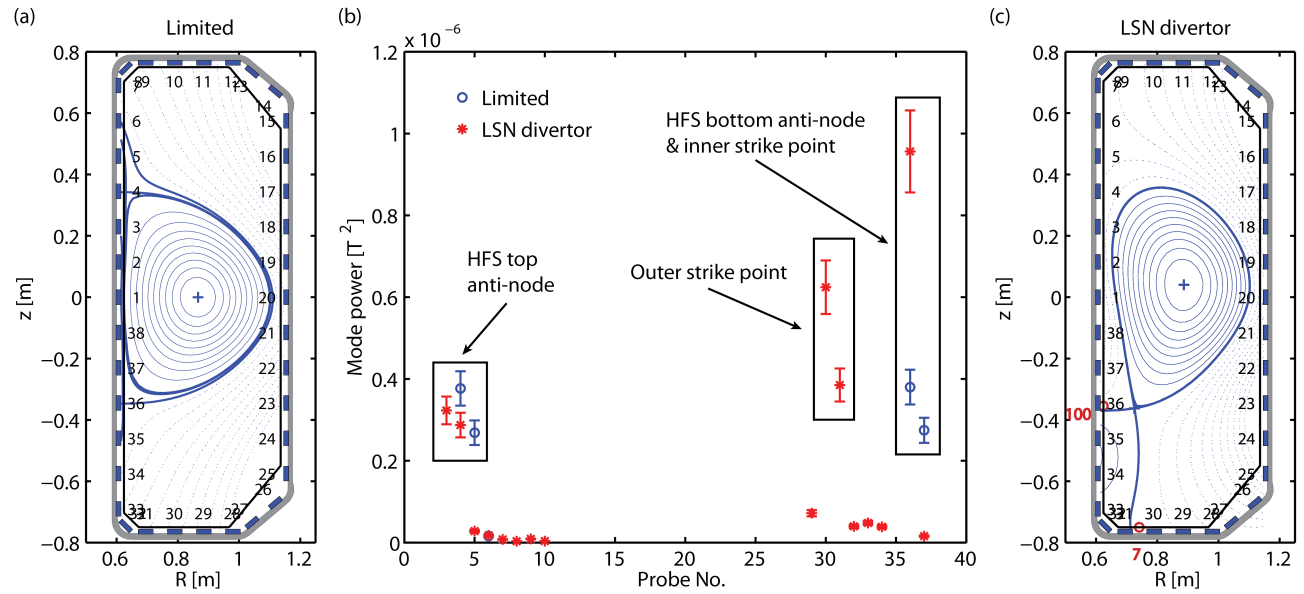

Figure 6. Plasma equilibrium of a limited \#49052 (a) and a LSN divertor \#52020 (c) TCV discharge. (b) GAM mode power on magnetic probes measured in these two discharges, calculated over a $100 \mathrm{~ms}$ time window with $1 \mathrm{kHz}$ frequency resolution, subtracted from background turbulence.

\subsection{Coherence and cross phase between different components}

The coherence and cross phase between these GAMs observed at the outer strike point and the conventional GAMs at the plasma edge inside LCFS is shown in Fig. 7 (a,b). The selected magnetic probe and Langmuir probe are both at the outer strike point 
(see Fig. 7 (c)), and the $\dot{\tilde{B}}$ and $\tilde{J}_{\text {i,sat }}$ components are found to be in phase. The TPCI signal is obtained at $\rho_{\psi}=0.97$; the coherence between this density component inside the LCFS and the SOL components at the GAM frequency is around 0.2. This value is well below what is usually measured in limited discharges between magnetic and TPCI signals (typically 0.8) [11], however the peak is clearly distinguishable from the background noise. The cross phase between $\tilde{n}$ and $\dot{\tilde{B}}$ is around $45^{\circ}$. High coherence is observed between the $D_{\alpha}$ emission signal and the magnetic component, and the cross phase is around $180^{\circ}$.
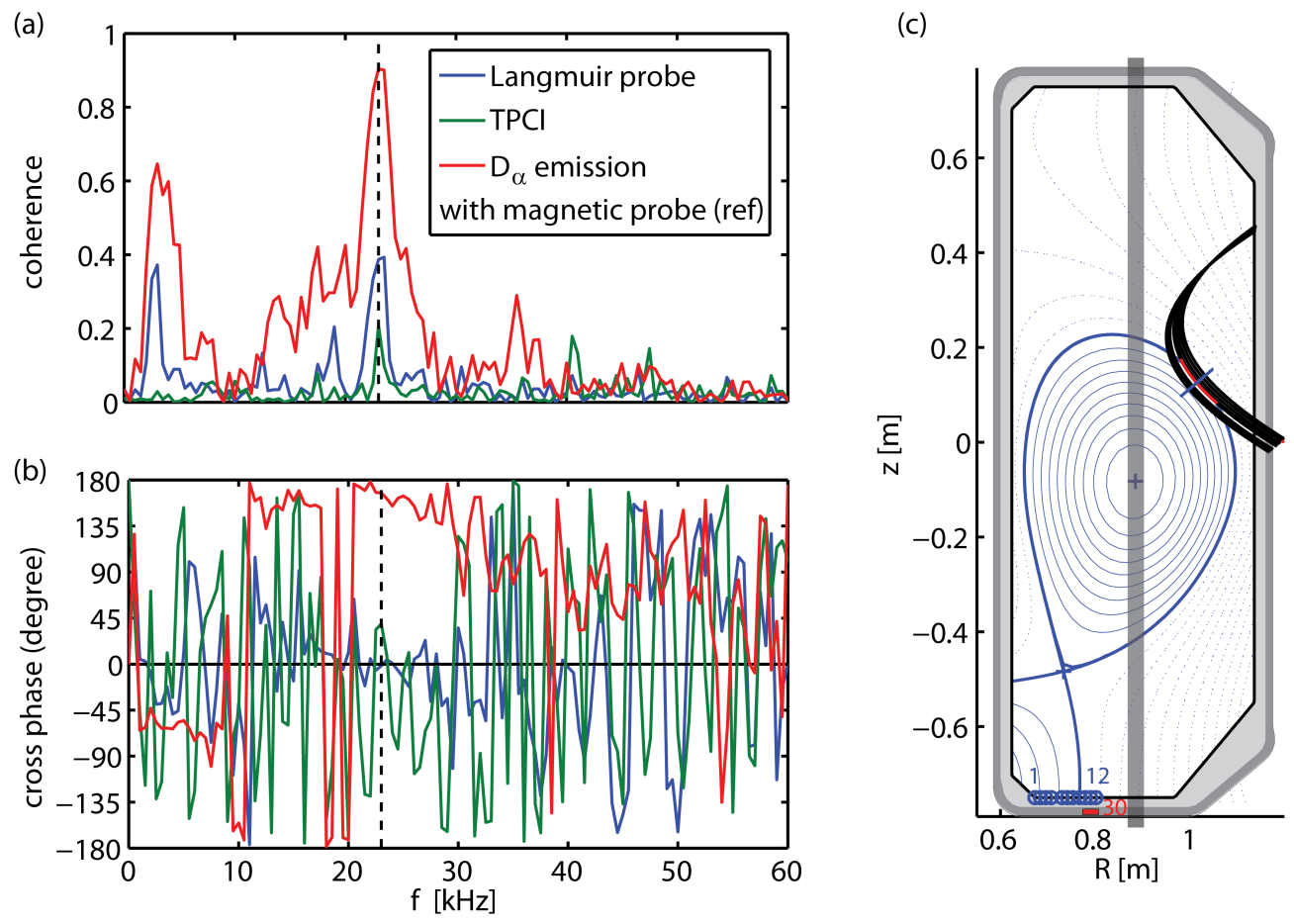

Figure 7. (a),(b) Squared coherence and cross phase of magnetic fluctuations (reference) and ion saturation current fluctuations at the outer strike point, electron density fluctuations at $\rho_{\psi}=0.97$ and $D_{\alpha}$ emission intensity fluctuations in TCV discharge 54490, calculated in a $40 \mathrm{~ms}$ time window with $0.5 \mathrm{kHz}$ frequency resolution. The black dashed line indicates the GAM peak at $23 \mathrm{kHz}$. (c) Plasma equilibrium and divertor configuration at $t=0.45 \mathrm{~s}$ in TCV discharge 54490. The black lines at the top right represent the poloidal projections of the TPCI laser chords, the red line highlight the chord used for analysis. The blue circles labeled from 1 to 12 represent the Langmuir probes, and the red square at $R=0.79 \mathrm{~m}$ labeled as 30 is the magnetic probe used for analysis. The gray rectangular box represents the vertical $D_{\alpha}$ line-of-sight.

\subsection{Mode distribution near strike points}

A strike point sweep was performed to study the localization of GAMs in the SOL, as shown in Fig. 8 (a). In this discharge, the outer strike point is swept from the HFS to the LFS during the LSN phase with nearly constant poloidal flux expansion $\left(f_{\mathrm{x}}=3-4\right)$. Here we focus on the time interval $t=0.4-0.5 \mathrm{~s}$ with $R_{\mathrm{SP}}$ sweeping 
from $0.685 \mathrm{~m}$ to $0.858 \mathrm{~m}$, through the Langmuir probe array from probe 1 to 19 . At $t=0.4,0.45$, and $0.5 \mathrm{~s}$, the strike point is roughly at the position of magnetic probes 31, 30 and 29, respectively. Fig. 8 (b) shows the spectrogram of signals from Langmuir probes $9-11$ at $t=0.43-0.47 \mathrm{~s}$ and Fig. 8 (c) shows the spectrogram of magnetic probes 31 to 29 at $t=0.35-0.55 \mathrm{~s}$. The GAM frequency is about $24 \mathrm{kHz}$. It can be seen from the spectrogram that the time range in which GAMs can be observed on a given Langmuir probe is around 10 to $20 \mathrm{~ms}$, while GAMs can last on a magnetic probe for more than $100 \mathrm{~ms}$ over the entire sweeping phase. The complete mode power profile at the target is reconstructed using all 19 Langmuir probes and 5 magnetic probes on the vessel floor, as shown in Fig. 8 (d) and (e) for the $\tilde{J}_{\mathrm{i} \text {,sat }}$ and $\tilde{B}$ components respectively. The distribution of the $\tilde{J}_{\mathrm{i}, \mathrm{sat}} \mathrm{GAM}$ mode power on the divertor target is very similar to the shape of the overall $\tilde{J}_{\mathrm{i} \text {,sat }}$ distribution, which peaks about $1.2 \mathrm{~cm}$ outside the strike point in the common flux region with a full width half maximum (FWHM) of $3.7 \mathrm{~cm}$; therefore it's almost absent in the private flux region. The target distribution of $\tilde{B}$ has a much larger FWHM $(8 \mathrm{~cm})$ and peaks inside the strike point $(-1.15 \mathrm{~cm})$, so it can be observed in both the common and the private flux region. Note that after $t=0.5 \mathrm{~s}$ when the outer strike point is on the LFS inclined wall structure, the GAM mode power vanishes from the Langmuir and magnetic probe measurements, which is not reflected in the single curve shown in Fig. 8 (d) and (e). In addition to the dependence of the magnetic GAM signal on the location relative to the SP, there is a therefore also an overall decrease in signal as the SP moves towards to the LFS.

In a LSN flaring experiment, illustrated by Fig. 9, the amplitude of the $\tilde{B}$ component of the GAMs is found to increase with the poloidal flux expansion $f_{\mathrm{x}}$. The outer strike point is slowly swept towards the HFS away from magnetic probe 30 from $t=0.5$ to $1 \mathrm{~s}$, with the distance between the two increasing from 9 to $17 \mathrm{~cm}$ during this time. However, the amplitude of the GAM increases during this sweep as shown in Figs. 9 (b) and (c), instead of decreasing as one would expect from Fig. 8 (e). This suggests that the distribution of the SOL $\tilde{B}$ component is affected by other parameters such as indeed the flux expansion. Fig. 9 (a) illustrates that although the strike point is moving away, the nearest flux surface to the magnetic probe 30 (within the resolution of the plot) is actually closer to the LCFS at $t=1 \mathrm{~s}$ than at $t=0.5 \mathrm{~s}$, due to the effect of flux expansion. Therefore instead of the radial distance from the probe to the strike point, the radial distance at the outer midplane (upstream) from LCFS to the nearest flux surface to the probe is used for analysis, as shown in Fig. 9 (d). The mode power profile peaks on the SOL flux surface $1.5 \mathrm{~cm}$ outside LCFS with a very narrow $0.69 \mathrm{~cm}$ FWHM, and drops to nearly zero before reaching the LCFS. This contradicts with the observation that the SOL $\tilde{B}$ component peaks at the target as shown in Fig. 8 (e). Note that the curve in Fig. 8 (e) includes only data from a discharge with nearly constant flux expansion $\left(f_{\mathrm{x}}=3-4\right)$. Therefore the GAM mode target distribution is modified by the flux expansion, through mechanisms that are not understood at present and may be elucidated by future experiments.

The studies of GAMs in the SOL were also extended to snowflake (SF) divertor and Super-X divertor discharges in TCV. The $\tilde{J}_{\mathrm{i}, \text { sat }}$ component of the GAM can be observed on the inner primary strike point of the snowflake-minus (SF-) configuration. This result is mainly consistent with the heat flux reduction at the target from SN to SF configurations in TCV [23]: GAMs are only detectable on the strike points on which the heat flux and particle flux remain high in going from the SN to the SF di- 

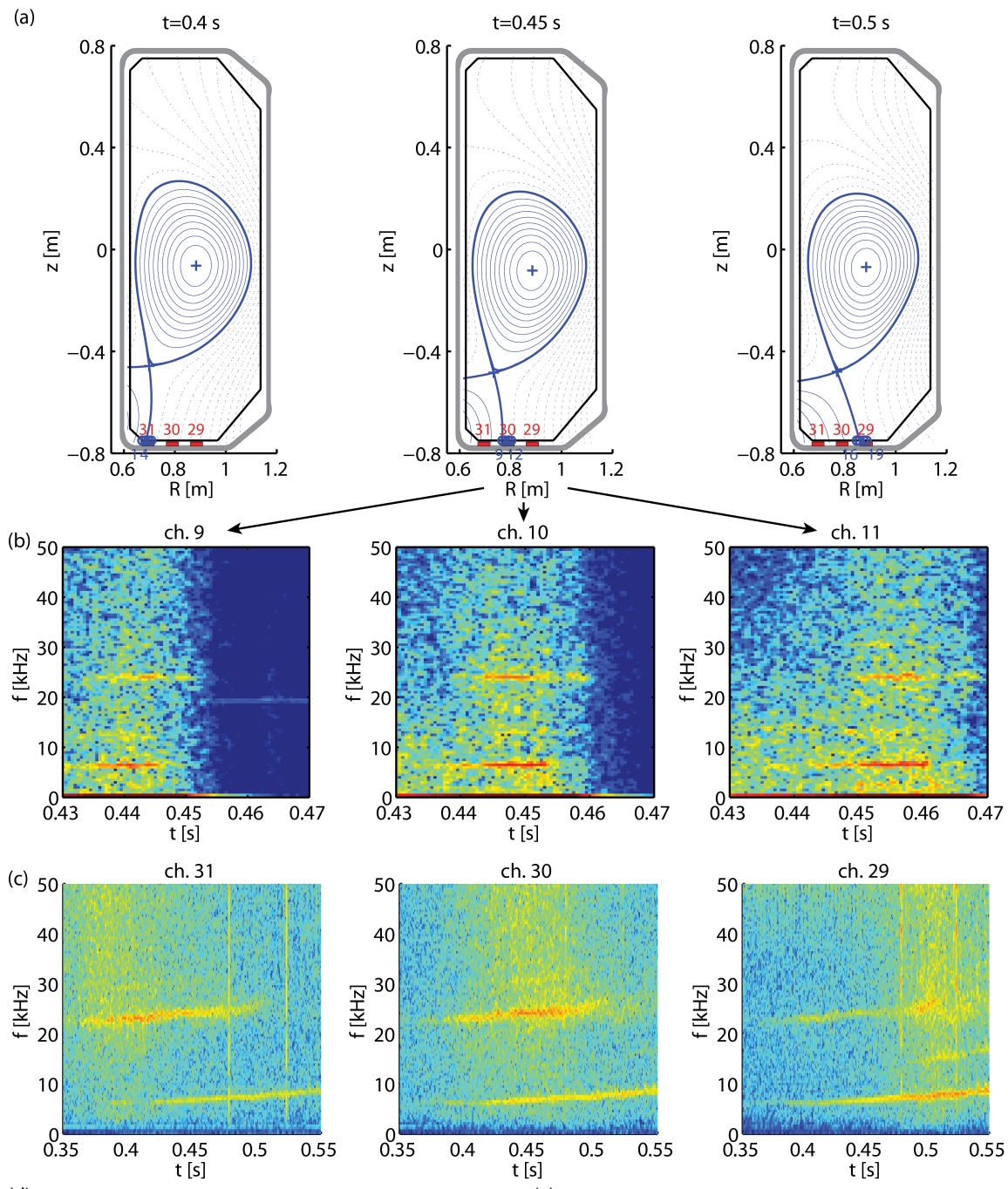

(d)
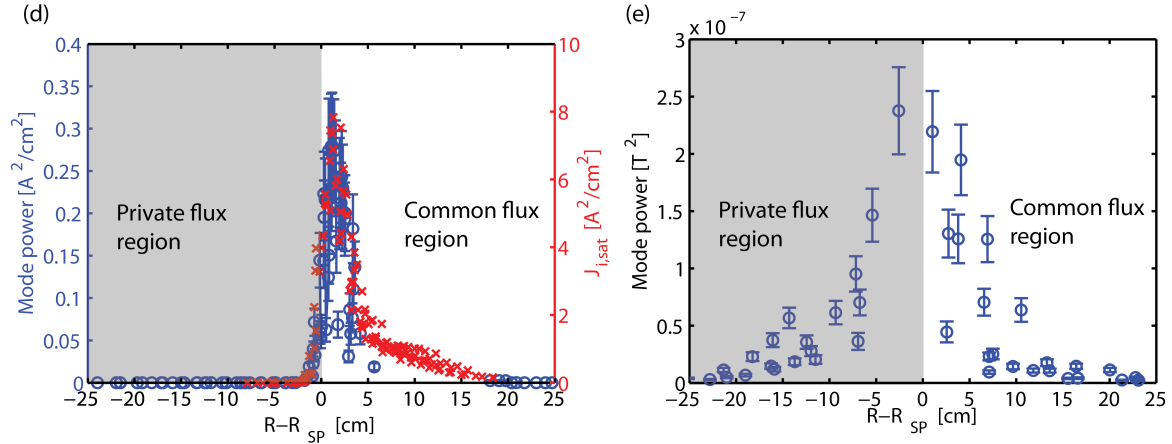

Figure 8. (a) Plasma equilibrium and divertor configuration at $t=0.4,0.45$ and $0.5 \mathrm{~s}$ in TCV discharge 54490. Langmuir probes and magnetic probes are represented in the same way as in Fig. 7. (b),(c) Auto-power spectrograms of ion saturation current fluctuations from Langmuir probes 9 to 11 and of magnetic fluctuations from magnetic probes 31 to 29. Distribution of GAM mode autopower component on (d) Langmuir probe and (e) magnetic probe signals along the target as a function of radial distance to the outer strike point; the mode power is integrated from 20 to $30 \mathrm{kHz}$ in a $28 \mathrm{~ms}$ time window with $0.5 \mathrm{kHz}$ frequency resolution. The red crosses indicate the total ion saturation current distribution (i.e., integrated over all frequencies). The shaded region indicates the private flux region. 
(a)
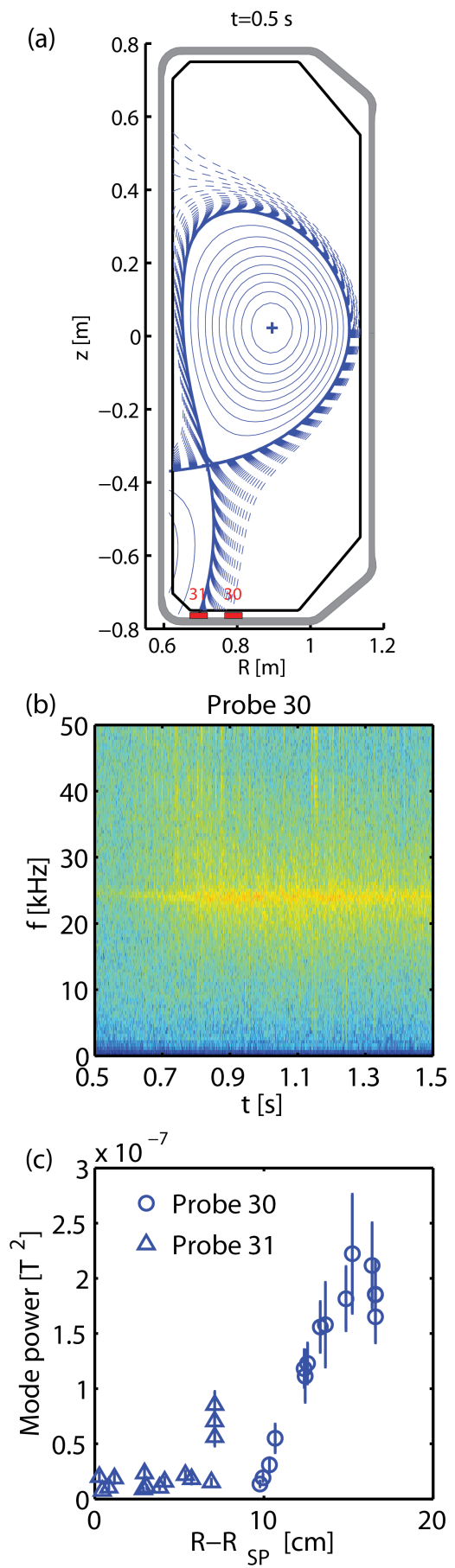
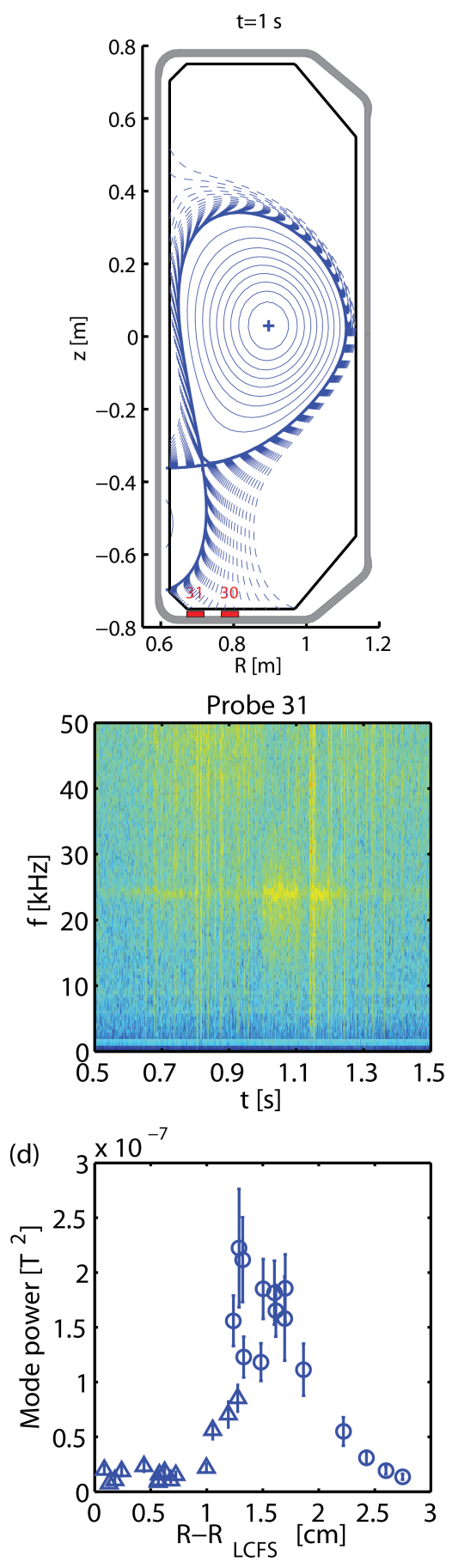

Figure 9. (a) Plasma equilibrium (solid curves) and SOL open flux surfaces (dashed curves) in TCV discharge 54816 at $t=0.5$ and $1 \mathrm{~s}$ with low and high poloidal flux expansion, respectively. The magnetic probes are represented in the same way as in Fig. 7. (b) Auto-power spectrograms of magnetic fluctuations from magnetic probes 30 and 31. Distribution of GAM mode power on magnetic probes 30 and 31 as a function of (c) radial distance to the outer strike point, and (d) distance to LCFS at midplane. The mode power is integrated from 20 to $30 \mathrm{kHz}$ over a $28 \mathrm{~ms}$ time window with $0.5 \mathrm{kHz}$ frequency resolution. 
vertor configuration. The magnetic component, as shown in Fig. 10, can be detected over a broader range as expected: in the SF- configuration (Fig. 10(a)), the GAM is detectable around both primary SPs at probes 31 and 35, as well as the bottom secondary SP around probe 29 ; in the SF + configuration, it is mainly localized around the inner primary SP at probe 35 and the bottom secondary SP at probe 29; in the Super-X divertor configuration, it is located from the inner SP to probe 29 in both LSN and Super-X configurations. The absence of GAMs at the outer primary strike point in the SF+ and Super-X configuration is consistent with the fact that the mode power tends to vanish as the outer strike point moves towards the LFS even for a SN configuration, as stated earlier in this paper.

The observations of GAM oscillations on the Langmuir probe signals suggest that a particle flux modulated at the GAM frequency travels along the SOL field lines to the strike points. The radial distribution of the $J_{\mathrm{i}, \text { sat }}$ component is consistent with the heat flux distribution. This hypothesis would also explain the GAM oscillations on the $D_{\alpha}$ emission intensity, as the particle flux hits the divertor target inducing recycling at the GAM frequency. The very fact that fluxes are observed to be modulated at the same frequency as the coherent mode observed in the core suggests a coupling between the two phenomena. Nothing that linear GAM physics alone cannot explain radial transport, a possible but still tentative explanation for the flux modulation is based on global gyrokinetic simulations using the GENE and ORB5 [28] codes, in which particle and heat fluxes modulated at the coherent frequency were observed, exhibiting avalanche-like behavior [1, 28, 29]. So far, no non-Gaussian probability distribution function (PDF) of electron density fluctuations has been obtained from TPCI observations. We note, however, that this is not incompatible with avalanche processes, in which a non-Gaussian PDF of heat fluxes can be obtained with Gaussian PDFs of electron temperature and density fluctuations. Also as the X-point topology and associated physics is not implemented in GENE or ORB5, the predictions were for limited plasmas, a configuration in which we should stress that we haven't observed any GAM signal on any SOL diagnostics such as inner wall Langmuir probes or $D_{\alpha}$ photodiodes. Further studies should be carried out in the future on the relation between GAM and avalanches.

\section{Conclusions}

In conclusion, GAMs have been further investigated in experiments in the TCV tokamak. A GAM regime transition from continuum to single-frequency radially extended regime was observed and analyzed in an ECH limited L-mode safety factor scan. Comparisons with global nonlinear gyrokinetic simulations suggest that the different electron density and temperature as well as their gradient profiles are the underlying reason for the two different regimes.

In addition, GAM oscillations have been observed and characterized on the SOL diagnostics near the strike points in various divertor configurations. This is the first study of its kind, even though oscillations at the edge GAM frequency were occasionally detected before by, e.g., $D_{\alpha}$ measurements in Globus-M [17] and Langmuir probe measurements in Alcator C-Mod [18]. An extensive characterization has been provided in varying divertor geometries. The results suggest a modulated particle flux that is qualitatively consistent with gyrokinetic simulations. Its underlying physical mechanism remains to be understood and may be related to avalanche phenomena. 
(a)

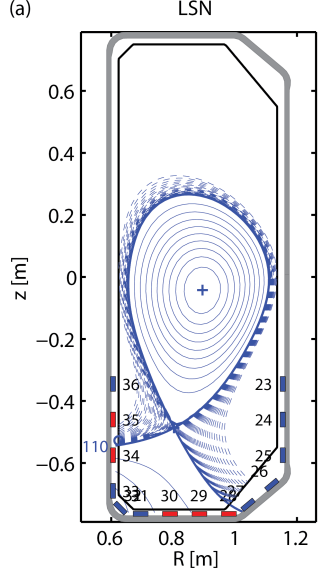

(b)
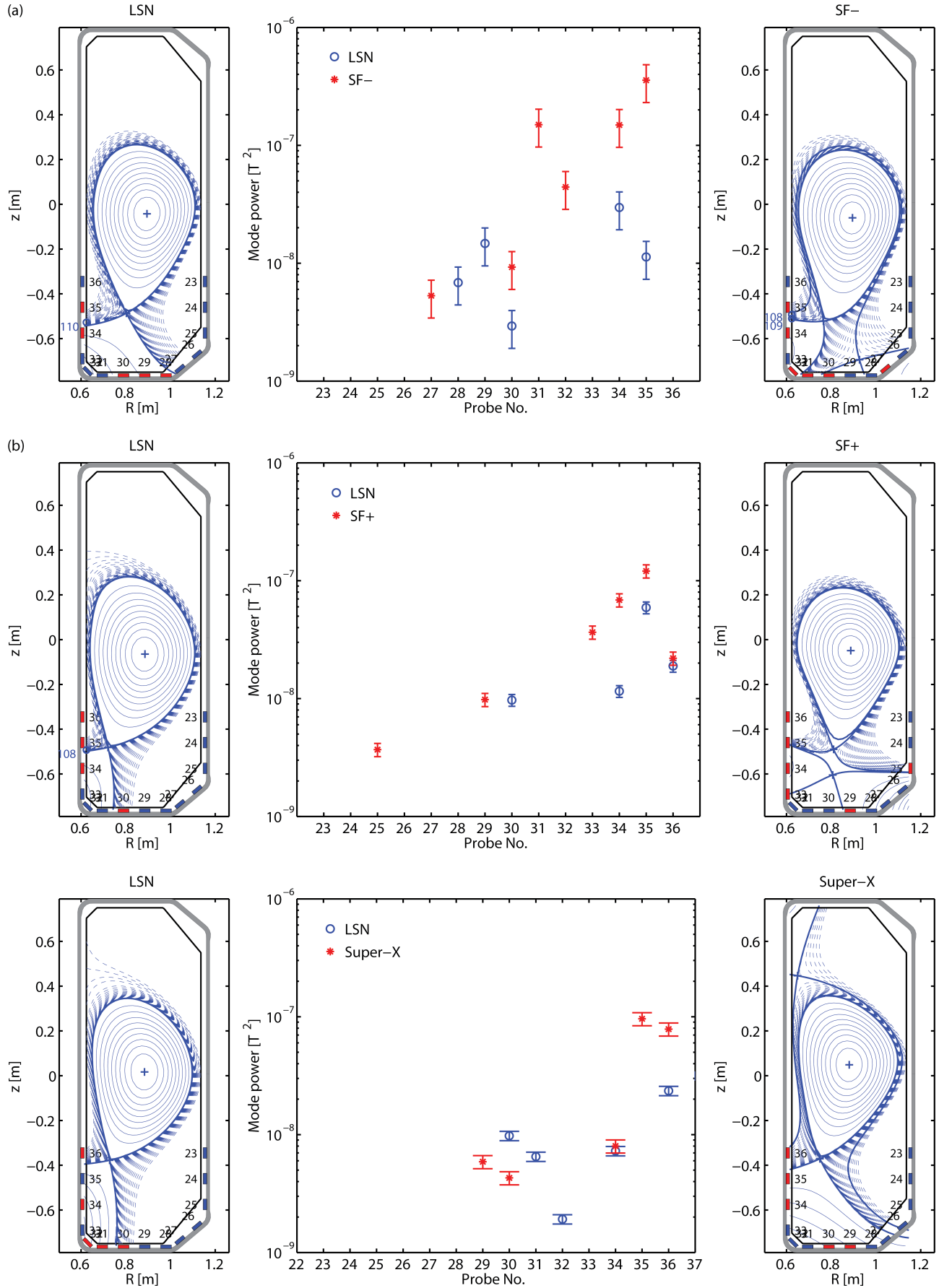

Figure 10. (Right) Plasma equilibria of a SF- \#54350 (a), a SF+ \#54378 (b) and a Super-X \#54308 TCV discharge; (Left) equilibria of LSN phases of the same discharges. The red squares highlight the magnetic probes on which the GAM is detectable; the blue circles represent the Langmuir probes on which the GAM is detectable. (Middle) GAM mode power on magnetic probes measured in these discharges, respectively. The blue circles indicate the LSN phase, the red stars indicate the SF/Super-X phase. The mode power is calculated by integrating the power spectrum over a $10 \mathrm{kHz}$ frequency range around the GAM frequency, and the spectrum is calculated over a $40 \mathrm{~ms}$ time window with $1 \mathrm{kHz}$ frequency resolution. 


\section{Acknowledgements}

The authors would like to thank H. Reimerdes, J. Graves and C. Wahlberg for valuable suggestions. Z. H. is grateful for fruitful discussions with K. Itoh and S-I. Itoh. This work has been carried out within the framework of the EUROfusion Consortium and has received funding from the Euratom research and training programme 20142018 under grant agreement No 633053. The views and opinions expressed herein do not necessarily reflect those of the European Commission. This work was also supported in part by the Swiss National Science Foundation and was partly performed in the framework of the Helmholtz Virtual Institute on Plasma Dynamical Processes and Turbulence Studies using Advanced Microwave Diagnostics.

\section{References}

[1] Merlo G, Brunner S, Huang Z, Coda S, Gorler T, Villard L, Banon Navarro A, Dominski J, Fontana M, Jenko F, Porte L and Told D 2017 Plasma Physics and Controlled Fusion this issue

[2] Diamond P H, Itoh S I, Itoh K and Hahm T S 2005 Plasma Physics and Controlled Fusion 47 R35-R161 ISSN 0741-3335 URL http://stacks . iop.org/0741-3335/47/i=5/a=R01?key=crossref . a4dceee0ba00403ce427a135a3569ce5

[3] Itoh K, Itoh S I, Diamond P H, Hahm T S, Fujisawa A, Tynan G R, Yagi M and Nagashima Y 2006 Physics of Plasmas 13055502 ISSN 1070-664X URL http://aip.scitation.org/doi/10.1063/1.2178779

[4] Winsor N 1968 Physics of Fluids 11 2448 URL http://scitation.aip.org/content/aip/journal/pof1/11/11/10.1063/1.1691835

[5] Conway G D, Tröster C, Scott B and Hallatschek K 2008 Plasma Physics and Controlled Fusion 50055009 URL http://stacks.iop.org/0741-3335/50/i=5/a=055009?key=crossref . 473584c468f28bca6933beb6a539fffa

[6] Angelino P, Garbet X, Villard L, Bottino A, Jolliet S, Ghendrih P, Grandgirard V, McMillan B F, Sarazin Y, Dif-Pradalier G and Tran T M 2008 Physics of Plasmas 15062306 URL http://aip.scitation.org/doi/10.1063/1.2928849

[7] Gao Z 2010 Physics of Plasmas $17 \quad 092503$ URL http://aip.scitation.org/doi/10.1063/1.3481464

[8] Fujisawa A 2009 Nuclear Fusion 49013001 URL http://stacks. iop.org/0029-5515/49/i=1/a=013001?key=crossref . 2af8915337ab3dc6d511dca6669ffb37

[9] Wang G, Peebles W A, Rhodes T L, Austin M E, Yan Z, McKee G R, La Haye R J, Burrell K H, Doyle E J, Hillesheim J C, Lanctot M J, Nazikian R, Petty C C, Schmitz L, Smith S, Strait E J, Van Zeeland M and Zeng L 2013 Physics of Plasmas 20092501 URL http://aip.scitation.org/doi/10.1063/1.4819501

[10] Ido T, Miura Y, Kamiya K, Hamada Y, Hoshino K, Fujisawa A, Itoh K, Itoh S I, Nishizawa A, Ogawa H, Kusama $\mathrm{Y}$ and Group J M 2006 Plasma Physics and Controlled Fusion 48 S41-S50 URL http://stacks. iop.org/0741-3335/48/i=4/a=S04?key=crossref . cbf6d541ba15b854c8ce8f98ecee55f3

[11] de Meijere C A, Coda S, Huang Z, Vermare L, Vernay T, Vuille V, Brunner S, Dominski J, Hennequin P, Krämer-Flecken A, Merlo G, Porte L 
and Villard L 2014 Plasma Physics and Controlled Fusion 56072001 URL http://stacks. iop.org/0741-3335/56/i=7/a=072001?key=crossref . 0365be6c61881eee82b09a481acec804

[12] Melnikov A, Eliseev L, Perfilov S, Lysenko S, Shurygin R, Zenin V, Grashin S, Krupnik L, Kozachek A, Solomatin R, Elfimov A, Smolyakov A and Ufimtsev M 2015 Nuclear Fusion 55063001 URL http://stacks . iop.org/0029-5515/55/i=6/a=063001?key=crossref . 9226cd58e576f2ca48d061730ae7b4e8

[13] Itoh K, Itoh S I, Diamond P H, Fujisawa A, Yagi M, Watari T, Nagashima $\mathrm{Y}$ and Fukuyama A 2006 Plasma and Fusion Research 1 037-037 URL http://joi.jlc.jst.go.jp/JST.JSTAGE/pfr/1.037?from=CrossRef

[14] Ilgisonis V I, Khalzov I V, Lakhin V P, Smolyakov A I and Sorokina E a 2014 Plasma Physics and Controlled Fusion 56035001 ISSN 0741-3335 URL http: //stacks . iop.org/0741-3335/56/i=3/a=035001?key=crossref . 91 fd94b836cd5837f45dfdc6af37638b

[15] Wahlberg $\mathrm{C}$ and Graves J $\quad$ P $2016 \quad$ Plasma Physics and Controlled Fusion $\mathbf{5 8} 075014 \quad$ URL http://stacks.iop.org/0741-3335/58/i=7/a=075014?key=crossref . c43dd4eb62f797e726baed4766b7db19

[16] Wahlberg C 2009 Plasma Physics and Controlled Fusion 51085006 URL http://stacks . iop.org/0741-3335/51/i=8/a=085006?key=crossref . b190e3a560bd0a4b4f c563109e20e6ae

[17] Bulanin V, Gusev V, Iblyaminova A, Khromov N, Kurskiev G, Minaev V, Patrov M, Petrov A, Petrov Y, Sakharov N, Shchegolev P, Tolstyakov S, Varfolomeev V, Wagner F and Yashin Y 2016 Nuclear Fusion 56016017 URL http://stacks.iop.org/0029-5515/56/i=1/a=016017?key=crossref .577b24dbf27e1fd4def6c79438f9052c

[18] Brunner D, Baek S, Hubbard A, Golfinopoulos T, Hughes J, Labombard B, Terry J L and Walk J 2015 Observations of low-frequency divertor fluctuations across confinement regimes in Alcator C-Mod Transport TaskForce meeting 2015

[19] Hofmann F, Lister J B, Anton W, Barry S, Behn R, Bernel S, Besson G, Buhlmann F, Chavan R, Corboz M, Dutch M J, Duval B P, Fasel D, Favre A, Franke S, Heym A, Hirt A, Hollenstein C, Isoz P, Joye B, Llobet X, Magnin J C, Marletaz B, Marmillod P, Martin Y, Mayor J M, Moret J M, Nieswand C, Paris P J, Perez A, Pietrzyk Z A, Pitts R A, Pochelon A, Rage R, Sauter O, Tonetti G, Tran M Q, Troyon F, Ward D J and Weisen H 1994 Plasma Physics and Controlled Fusion 36 B277-B287 URL http://stacks.iop.org/0741-3335/36/i=12B/a=023?key=crossref . 1b42d2ef 9fb94c7eeb837ce0e2c29250

[20] Coda S 2015 Nuclear Fusion 5504004 URL http://stacks.iop.org/0029-5515/55/i=10/a=104004?key=crossref. eb289508866094016711af3dd27e1c95

[21] Theiler C, Lipschultz B, Harrison J, Labit B, Reimerdes H, Tsui C, Vijvers W, Boedo J A, Duval B, Elmore S, Innocente P, Kruezi U, Lunt T, Maurizio R, Nespoli F, Sheikh U, Thornton A, van Limpt S, Verhaegh K and Vianello N 2017 Nuclear Fusion 57072008 ISSN 0029-5515 URL http://stacks.iop.org/0029-5515/57/i=7/a=072008?key=crossref . e0ba8c2b73c98effa804e1a3ad432e8c

[22] Piras F, Coda S, Furno I, Moret J M, Pitts R A, Sauter O, Tal B, Turri G, Bencze A, Duval B P, Felici F, Pochelon A and Zucca C 2009 Plasma Physics and Controlled Fusion 51055009 URL http://stacks.iop.org/0741-3335/51/i=5/a=055009?key=crossref . a281e046d21242eba91cedf c60587859

[23] Reimerdes H, Canal G P, Duval B P, Labit B, Lunt T, Vijvers W A J, Coda S, De Temmerman G, Morgan T W, Nespoli F and Tal B 2013 Plasma Physics and Controlled Fusion 55124027 URL http://stacks.iop.org/0741-3335/55/i=12/a=124027?key=crossref. bc4a3adf8acbb82cfb7f392553940e52 
[24] Marinoni A, Coda S, Chavan R and Pochon G 2006 Review of Scientific Instruments 77 10E929 URL http://aip.scitation.org/doi/10.1063/1.2222333

[25] Moret J M, Buhlmann F, Fasel D, Hofmann F and Tonetti G 1998 Review of Scientific Instruments $\mathbf{6 9} 2333$ URL http://aip.scitation.org/doi/10.1063/1.1148940

[26] Jenko F, Dorland W, Kotschenreuther M and Rogers B N 2000 Physics of Plasmas 71904 URL https://doi.org/10.1063/1.874014

[27] Rosenbluth M and Hinton F 1998 Physical Review Letters 80 724-727 ISSN 00319007

[28] McMillan B F, Jolliet S, Tran T M, Villard L, Bottino A and Angelino P 2009 Physics of Plasmas 16022310 ISSN 1070664X

[29] Villard L, McMillan B F, Sauter O, Hariri F, Dominski J, Merlo G, Brunner S and Tran T M 2014 Journal of Physics: Conference Series 561012022 URL http://stacks.iop.org/1742-6596/561/i=1/a=012022 